\title{
Determination of QCD phase diagram from the imaginary chemical potential
}

\author{
Yuji Sakai* \\ Department of Physics, Graduate School of Sciences, Kyushu University, \\ Fukuoka 812-8581, Japan \\ E-mail: sakaiephys.kyushu-u.ac.jp

\section{Kouji Kashiwa} \\ Department of Physics, Graduate School of Sciences, Kyushu University, \\ Fukuoka 812-8581, Japan \\ E-mail: kashiwa@phys.kyushu-u.ac.jp
}

\section{Hiroaki Kouno}

Department of Physics, Saga University,

Saga 840-8502, Japan

E-mail: kounohecc.saga-u.ac.jp

\section{Masanobu Yahiro}

Department of Physics, Graduate School of Sciences, Kyushu University,

Fukuoka 812-8581, Japan

E-mail: yahiro@phys.kyushu-u.ac.jp

Lattice QCD has the well-known sign problem at real chemical potential. One approach to circumvent the problem is the analytic continuation from imaginary chemical potential to real one. We propose a new analytic continuation by using the Polyakov-loop extended Nambu-JonaLasinio (PNJL) model. The partition function of QCD has the Roberge-Weiss (RW) periodicity in the imaginary chemical potential region. We reveal that the PNJL model has the RW periodicity. Strength parameters of the vector-type four-quark and scalar-type eight-quark interactions are determined so as to reproduce lattice data on pseudocritical temperatures of the deconfinement and chiral transitions in the imaginary chemical potential region. The QCD phase diagram in the real chemical potential region is predicted by the PNJL model. The critical endpoint survives, even if the vector-type four-quark interaction is taken into account.

The XXVII International Symposium on Lattice Field Theory - LAT2009

July 26-31 2009

Peking University, Beijing, China

\footnotetext{
* Speaker.
} 


\section{Introduction}

Quantum chromodynamics (QCD) is a remarkable theory. It is renormalizable and essentially parameter free. QCD accounts for the rich phenomenology of hadronic and nuclear physics. Thermodynamics of QCD is also well defined. Nevertheless, it is not well known because of its nonperturbative nature. In particular, the QCD phase diagram is essential for understanding not only natural phenomena such as compact stars and the early universe but also laboratory experiments such as relativistic heavy-ion collisions.

Unfortunately, quantitative calculations of the phase diagram from the first-principle lattice QCD (LQCD) have the well-known sign problem when the chemical potential $(\mu)$ is real. So far, several approaches have been proposed to circumvent the difficulty; for example, the reweighting method, the Taylor-expansion method, and the analytic continuation to real chemical potential $\left(\mu_{\mathrm{R}}\right)$ from imaginary chemical potential $\left(\mu_{\mathrm{I}}\right)[1]$. However, those are still far from perfection.

As an approach complementary to the first-principle LQCD, we can consider effective models such as the Nambu-Jona-Lasinio (NJL) model and the Polyakov-loop extended Nambu-JonaLasinio (PNJL) model $[2,3]$. The NJL model describes the chiral symmetry breaking, but not the confinement mechanism. The PNJL model can treat the deconfinement transition as well as the chiral symmetry restoration. In the NJL-type models, the input parameters are determined at $\mu=0$ and $T>0$, where $\mathrm{T}$ is temperature. It is then highly nontrivial whether the models predict properly dynamics of QCD at finite $\mu_{\mathrm{R}}$. This should be tested from QCD. Fortunately, this is possible in the $\mu_{\mathrm{I}}$ region, since $\mathrm{LQCD}$ has no sign problem there. Therefore, the reliability of effective models at finite $\mu_{\mathrm{R}}$ can be tested in the $\mu_{\mathrm{I}}$ region.

Roberge and Weiss found [4] that the QCD partition function $Z_{\mathrm{QCD}}(\theta)$ at imaginary chemical potential $\mu_{\mathrm{I}}=i \theta T$ has a periodicity $Z_{\mathrm{QCD}}(\theta)=Z_{\mathrm{QCD}}(\theta+2 \pi / 3)$, showing that $Z_{\mathrm{QCD}}(\theta+2 \pi / 3)$ is transformed into $Z_{\mathrm{QCD}}(\theta)$ by the $\mathbb{Z}_{3}$ transformation with integer $k$. This means that QCD is invariant under a combination of the $\mathbb{Z}_{3}$ transformation and a parameter transformation $\theta \rightarrow \theta+$ $2 \pi / 3$,

$$
q \rightarrow U q, A_{v} \rightarrow U A_{v} U^{-1}-\frac{i}{g}\left(\partial_{v} U\right) U^{-1}, \quad \theta \rightarrow \theta+2 \pi / 3,
$$

where $U(x, \tau)$ are elements of $S U(3)$ with $U(x, 1 / T)=\exp (-2 i \pi k) U(x, 0)$ and q is the quark field. We call this combination the extended $\mathbb{Z}_{3}$ transformation. Thus, $Z_{\mathrm{QCD}}(\theta)$ has the extended $\mathbb{Z}_{3}$ symmetry, and hence quantities invariant under the extended $\mathbb{Z}_{3}$ transformation have the RobergeWeiss (RW) periodicity. At the present stage, the PNJL model is only a realistic effective model that possesses both the extended $\mathbb{Z}_{3}$ symmetry and chiral symmetry [5]. This property guarantees that the phase diagram evaluated by the PNJL model has the RW periodicity in the imaginary $\mu_{\mathrm{I}}$ region, and therefore makes it possible to compare the PNJL model with LQCD quantitatively in the $\mu_{\mathrm{I}}$ region. If the PNJL model succeeds in reproducing the lattice data, we may think that the PNJL model will predict, with high reliability, the QCD phase structure in the $\mu_{\mathrm{R}}$ region [5].

The extended $\mathbb{Z}_{3}$ symmetry in QCD is a remnant of the $\mathbb{Z}_{3}$ symmetry, namely the confinement mechanism, in the pure gauge system. The extended $\mathbb{Z}_{3}$ symmetry appears as the RW periodicity in the $\mu_{\mathrm{I}}$ region and implicitly affects dynamics in the $\mu_{\mathrm{R}}$ region. Actually, the mechanism largely shifts the critical endpoint toward higher $T$ and lower $\mu$ than the NJL model predicts. 
In contrast, the vector-type four-quark interaction $G_{\mathrm{v}}\left(\bar{q} \gamma_{v} q\right)^{2}$ largely moves the critical endpoint in the opposite direction, if it is newly added to the PNJL model. Thus, it is essential to determine the strength of the coupling $G_{\mathrm{v}}$ of the vector-type interaction, although the interaction is often ignored in the PNJL calculations. The strength of $G_{\mathrm{v}}$ can be determined from lattice data on the chiral transition in the $\mu_{\mathrm{I}}$ region [5].

\section{PNJL Model}

The two-flavor PNJL Lagrangian in Euclidean spacetime is

$$
\mathscr{L}=\bar{q}\left(i \gamma_{v} D_{v}-\gamma_{4} \mu+m_{0}\right) q-G_{\mathrm{s}}\left[(\bar{q} q]^{2}+\left(\bar{q} i \gamma_{5} \vec{\tau} q\right)^{2}\right]+U_{\mathrm{p}}\left(\Phi[A], \Phi^{*}[A], T\right),
$$

where $q$ denotes the two-flavor quark field, $m_{0}$ does the current quark mass, and $D_{v}=\partial_{v}-i A_{v}$, $A_{v}=g A_{4}^{a} \frac{\lambda_{a}}{2} \delta_{v 4}$ with the gauge field $A_{v}^{a}$, the Gell-Mann matrix $\lambda^{a}$, and the gauge coupling $\mathrm{g}$. In the NJL sector, $G_{\mathrm{s}}$ denotes the coupling constant of the scalar-type four-quark interaction. Later, we will add the vector-type four-quark interaction and the scalar-type eight-quark interaction to the PNJL Lagrangian [5]. The Polyakov potential $U_{\mathrm{p}}$, defined in (2.4), is a function of the Polyakov loop $\Phi$ and its Hermitian conjugate $\Phi^{*}$,

$$
\Phi=\frac{1}{N_{\mathrm{c}}} \operatorname{Tr}_{\mathrm{c}} L, \Phi^{*}=\frac{1}{N_{\mathrm{c}}} \operatorname{Tr}_{\mathrm{c}} L^{\dagger}, \text { with } L(\mathbf{x})=\mathscr{P} \exp \left[i \int_{0}^{1 / T} d \tau A_{4}(\mathbf{x}, \tau)\right]
$$

where $\mathscr{P}$ is the path ordering. In the chiral limit $\left(m_{0}=0\right)$, the Lagrangian density has the exact $S U\left(N_{f}\right)_{\mathrm{R}} \times S U\left(N_{f}\right)_{\mathrm{L}} \times U(1)_{\mathrm{v}} \times S U(3)_{\mathrm{c}}$ symmetry. In the Polyakov gauge, $L$ can be written in a diagonal form in color space $L=\operatorname{diag}\left(e^{i \phi_{a} / T}, e^{i \phi_{b} / T}, e^{i \phi_{c} / T}\right)$. The Polyakov loop $\Phi$ is an exact order parameter of the spontaneous $\mathbb{Z}_{3}$ symmetry breaking in the pure gauge theory. Although the $\mathbb{Z}_{3}$ symmetry is not exact in the system with dynamical quarks, it still seems to be a good indicator of the deconfinement transition. Therefore, we use $\Phi$ to define the deconfinement transition.

Making the mean field approximation and performing the path integral over quark field, one can obtain the thermodynamic potential $\Omega$ with imaginary $\mu_{\mathrm{I}}$,

$$
\Omega=U_{\mathrm{p}}+U_{\mathrm{M}}-2 \sum_{f} \int \frac{d^{3} \mathbf{p}}{(2 \pi)^{3}}\left[N_{\mathrm{c}} \varepsilon_{f}(\mathbf{p})+T \sum_{\lambda= \pm 1} \ln \operatorname{det}_{\mathrm{c}}\left(1+L^{\lambda} e^{-\left(\varepsilon_{f}(\mathbf{p})-i \lambda \mu_{\mathrm{I}}\right) / T}\right)\right],
$$

where $\varepsilon_{f}=\sqrt{\mathbf{p}^{2}+M_{f}^{2}}, M_{f}=m_{0}-2 G_{\mathrm{s}} \sigma_{f}, U_{\mathrm{M}}=G_{\mathrm{s}} \sigma_{f}^{2}$ and $\sigma_{f}=\left\langle\overline{q_{f}} q_{f}\right\rangle$. In this case, we consider two degenerate flavors, so all $\sigma_{f}$ are degenerated $\left(\sigma_{f}=\sigma\right)$. In (2.3), only the third term of the right-hand side diverges. It is then regularized by the three dimensional momentum cutoff $\Lambda$. We use $U_{\mathrm{p}}$ of Ref. [3] that is fitted to a LQCD simulation in the pure gauge theory at finite T:

$$
\begin{gathered}
U_{\mathrm{p}}=T^{4}\left[-\frac{a(T)}{2} \Phi^{*} \Phi+b(T) \ln \left[1-6 \Phi^{*} \Phi+4\left(\Phi^{3}+\Phi^{3}\right)-3\left(\Phi^{*} \Phi\right)^{2}\right]\right], \\
a(T)=3.51-2.47\left(\frac{T_{0}}{T}\right)+15.2\left(\frac{T_{0}}{T}\right)^{2}, \quad b(T)=-1.75\left(\frac{T_{0}}{T}\right)^{3} .
\end{gathered}
$$

The Polyakov potential yields a first-order deconfinement phase transition at $T=T_{0}$ in the pure gauge theory. The original value of $T_{0}$ is $270 \mathrm{MeV}$ evaluated by the pure gauge LQCD calculation. 
However, the PNJL model with this value of $T_{0}$ yields a somewhat larger value of the transition temperature at zero chemical potential than the full LQCD simulation [6]. Therefore, we rescale $T_{0}$ to $212 \mathrm{MeV}$; the detail will be shown in Sec. 3.1.

The PNJL thermodynamic potential $\Omega$ of (2.3) is invariant under the extended $\mathbb{Z}_{3}$ transformation,

$$
L \rightarrow e^{-i 2 \pi k / 3} L, \quad \theta=\mu_{\mathrm{I}} / T \rightarrow \theta+2 \pi k / 3,
$$

therefore $\Omega$ has the RW periodicity. The left panel of Fig. 1 shows the PNJL thermodynamic potential $\Omega$ as a function of $\theta$ in two cases of $T=170$ and $190 \mathrm{MeV}$. The potential $\Omega$ is smooth everywhere in the low $T$ case, but not at $\theta=(2 k+1) \pi / 3$ in the high $T$ case. This result is consistent with the RW prediction [4] and lattice simulation [1] on the $\theta$ and the $T$ dependence of the QCD thermodynamic potential. Furthermore, we can define the extended $\mathbb{Z}_{3}$ invariant quantities $\Psi=e^{-i \theta} \Phi$ called $\Psi$ as the modified Polyakov loop.
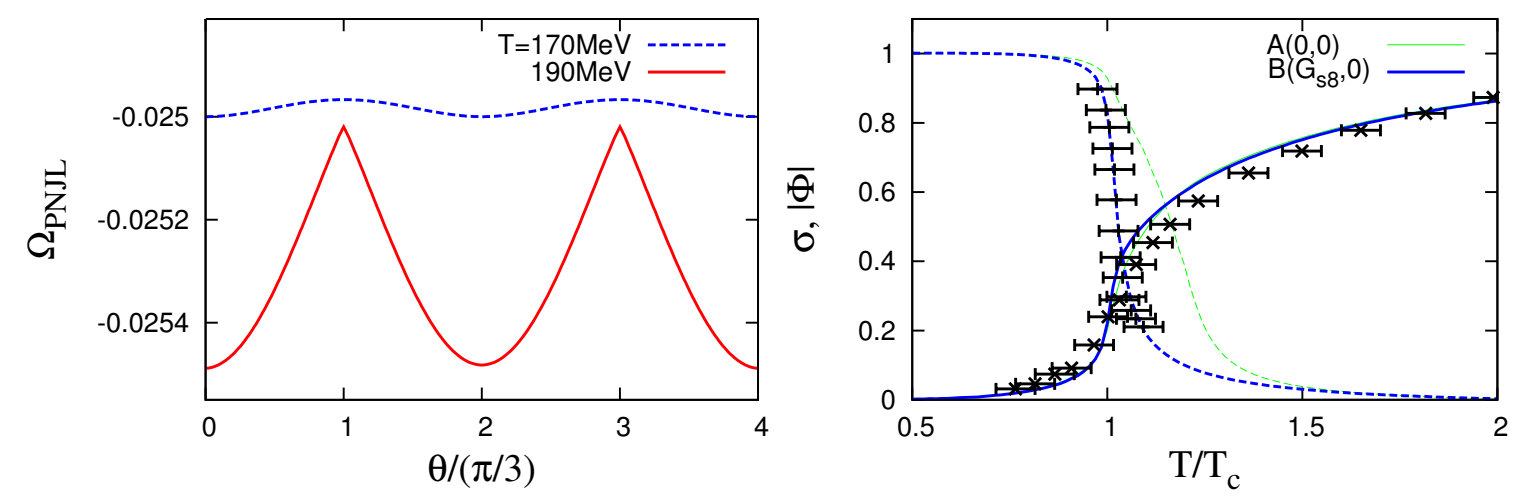

Figure 1: Left panel; The PNJL thermodynamic potential $\Omega$ as a function of $\theta$. The dashed line corresponds to the case of $T=170 \mathrm{MeV}$ and the solid one to that of $T=190 \mathrm{MeV}$. Right panel; Chiral condensate normalized by $\sigma_{0}$ and the absolute value of the Polyakov loop $|\Phi|$. The thick (thin) curves represent the PNJL result of parameter set B (A) with (without) the scalar-type eight-quark interaction; $\sigma(|\Phi|)$ is denoted by the dashed (solid) curves. Lattice data ( + ) on $\sigma$ and those ( $\times$ ) on $|\Phi|$ are taken from Ref. [6]. The lattice data are plotted with a $10 \%$ error bar, since lattice calculations have $10 \%$ error in determining $T_{\mathrm{c}}[6]$.

\section{Comparision between PNJL and LQCD}

\subsection{Thermal system with no chemical potential}

First, we consider the thermal system with no chemical potential to determine the parameters, $m_{0}, G_{\mathrm{s}}, \Lambda$, and $T_{0}$, of the PNJL model. In the lattice calculations [6], the pseudocritical temperature $T_{\mathrm{c}}^{\sigma}$ of the crossover chiral transition coincides with that $T_{\mathrm{c}}^{\Phi}$ of the crossover deconfinement one within $10 \%$ error: $T_{\mathrm{c}}^{\sigma} \approx T_{\mathrm{c}}^{\Phi} \approx 173 \pm 8 \mathrm{MeV}$.

The parameter set, $\Lambda=631.5 \mathrm{MeV}, G_{\mathrm{s}}=5.498 \mathrm{GeV}^{-2}$, and $m_{0}=5.5 \mathrm{MeV}$, can reproduce the pion decay constant $f_{\pi}=93.3 \mathrm{MeV}$ and the pion mass $M_{\pi}=138 \mathrm{MeV}$ at $T=\mu=0$, and keeps a good reproduction LQCD data at finite $T$ [3]. We then adopt these values for $\Lambda, G_{\mathrm{s}}$, and $m_{0}$. We adjust $T_{0}$ so that the PNJL calculation can reproduce the lattice result $T_{\mathrm{c}}^{\Phi}=173 \mathrm{MeV}$. The parameter set thus determined is shown as set A in Table 1. 
The right panel of Fig. 1 shows the chiral condensate normalized by $\sigma_{0}=\left.\sigma\right|_{T=\mu=0}$ and the absolute value of the Polyakov loop $\Phi$ as a function of $T / T_{\mathrm{c}}$. In this paper $T_{\mathrm{c}}$ is always taken to be $173 \mathrm{MeV}$. The thin curves represent PNJL results of parameter set A, where $\sigma_{0}=-0.0302 \mathrm{GeV}^{3}$ in this case. LQCD data [6] are also plotted by cross symbols with a $10 \%$ error bar; $\sigma$ and $|\Phi|$ measured as a function of $T / T_{\mathrm{c}}$ in Refs. [6] have only small errors, but we have added $10 \%$ error that the lattice calculation [6] has in determining $T_{\mathrm{c}}$. For $|\Phi|$, the PNJL result (thin solid curve) reasonably agrees with the lattice one $(\times)$. For $\sigma$, however, the PNJL result (thin dashed curve) considerably overshoots the lattice data (+). The PNJL results of parameter set A give $T_{\mathrm{c}}^{\sigma} / T_{\mathrm{c}}=$ 1.25 and $T_{\mathrm{c}}^{\Phi} / T_{\mathrm{c}}=1$, while the lattice simulations yield $T_{\mathrm{c}}^{\sigma} / T_{\mathrm{c}}=1 \pm 0.05$ and $T_{\mathrm{c}}^{\Phi} / T_{\mathrm{c}}=1 \pm 0.05$. The PNJL results are consistent with the lattice ones for $T_{\mathrm{c}}^{\Phi}$, but not for $T_{\mathrm{c}}^{\sigma}$.

In order to solve this problem, we introduce the scalar-type eight-quark interaction, $G_{\mathrm{s} 8}\left[(\bar{q} q)^{2}+\right.$ $\left.\left(\bar{q} i \gamma_{5} \vec{\tau} q\right)^{2}\right]^{2}[5]$. Since $f_{\pi}$ and $M_{\pi}$ calculated with PNJL depend on the strength of $G_{\mathrm{s} 8}$, for each value of $G_{\mathrm{s} 8}$ the strength of $G_{\mathrm{s}}$ is readjusted so as to reproduce the measured values $f_{\pi}=93.3 \mathrm{MeV}$ and $M_{\pi}=138 \mathrm{MeV}$. As $G_{\mathrm{s} 8}$ increases from zero, $T_{\mathrm{c}}^{\sigma}$ calculated with PNJL decreases toward $T_{\mathrm{c}}=173$ $\mathrm{MeV}$. When $G_{\mathrm{s} 8}=452.12 \mathrm{GeV}^{-8}$, the ratio $T_{\mathrm{c}}^{\sigma} / T_{\mathrm{c}}$ becomes 1.05 and hence, consistent with the corresponding lattice result within $10 \%$ error. We adopt this strength. This parameter set is shown as set $\mathrm{B}$ in Table 1 .

\subsection{Thermal system with imaginary chemical potential}

In this subsection, we consider the thermal system with finite imaginary chemical potential and compare the PNJL result with the lattice data [1] in which the lattice size is $8^{3} \times 4$ and the two-flavor Kogut-Susskind and Wilson fermions are considered.

The left panel of Fig. 2 shows the phase diagram of the chiral and deconfinement transitions in the $\theta-T$ plane, where $\theta$ is divided by $\pi / 3$ and $T$ is normalized by $T_{\mathrm{c}}=173 \mathrm{MeV}$. The lattice data [1] are shown with $10 \%$ error that $T_{\mathrm{c}}$ has [6]. The lattice simulations [1] point out that $T_{\mathrm{c}}^{\sigma}$ agrees with $T_{\mathrm{c}}^{\Phi}$ within numerical errors in the entire region $0 \leq \theta \leq 2 \pi / 3$. Thin, thick, and bold curves are results of the PNJL calculations with sets $\mathrm{A}, \mathrm{B}$, and $\mathrm{C}$, respectively.

\begin{tabular}{cccc}
\hline set & $G_{\mathrm{s}}$ & $G_{\mathrm{s} 8}$ & $G_{\mathrm{v}}$ \\
\hline A & $5.498 \mathrm{GeV}^{-2}$ & 0 & 0 \\
\hline B & $4.673 \mathrm{GeV}^{-2}$ & $452.12 \mathrm{GeV}^{-8}$ & 0 \\
\hline $\mathrm{C}$ & $4.673 \mathrm{GeV}^{-2}$ & $452.12 \mathrm{GeV}^{-8}$ & $4.673 \mathrm{GeV}^{-2}$ \\
\hline
\end{tabular}

Table 1: Summary of the parameter sets in the PNJL calculations. The parameters $\Lambda, m_{0}$ and $T_{0}$ are common among the three sets; $\Lambda=631.5 \mathrm{MeV}, m_{0}=5.5 \mathrm{MeV}$ and $T_{0}=212 \mathrm{MeV}$.

The phase diagram has a periodicity of $2 \pi / 3$ in $\theta$. This is called the RW periodicity [4,5]. The phase diagram is also $\theta$ even, because so is $\Omega$. On the dot-dashed line going up from an endpoint $\left(\theta_{\mathrm{RW}}, T_{\mathrm{RW}}\right)=\left(\pi / 3,1.09 T_{\mathrm{c}}\right)$, the quark-number density $n$ and the phase $\phi$ of the Polyakov loop are discontinuous in the PNJL calculations [5]. This is called the RW phase transition. The right panel of Fig. 2 shows $\theta$ dependence of $\phi$ for four cases of $T / T_{\mathrm{c}}=0.97,1.01,1.04$, and 1.10. The PNJL results (curves) well reproduce the lattice data [1] (symbols). It is found that is continuous at $\theta=\pi / 3$ in the low- $\mathrm{T}$ case of $T \leq T_{\mathrm{RW}}=1.09 T_{\mathrm{c}}$, but it is discontinuous at $\theta=\pi / 3$ in the high- $\mathrm{T}$ case of $T>T_{\mathrm{RW}}$. 
In the entire region $0 \leq \theta \leq 2 \pi / 3$, the eight-quark interaction moves $T_{\mathrm{c}}^{\sigma}$ down from the thin dashed curve (set A) to the thick one (set B). However, the thick dashed curve still overshoots the lattice result (symbols) with $10 \%$ error near $\theta=\pi / 3$. In order to solve this problem, we introduce the vector-type four-quark interaction, $-G_{\mathrm{v}}\left(\bar{q} \gamma_{v} q\right)^{2}$ [5]. As mentioned in Ref. [5], the phase structure in the real chemical potential region is quite sensitive to the strength of the coupling $G_{\mathrm{v}}$. It is then important to determine the strength, but it has not been done yet. Since the vector-type interaction does not affect the physical quantities at $\mu=0$, we can simply add the interaction to set B. As $G_{\mathrm{v}}$ increases from zero, $T_{\mathrm{c}}^{\sigma}$ goes down toward $T_{\mathrm{c}}^{\Phi}$, while $T_{\mathrm{c}}^{\Phi}$ moves little. When $G_{\mathrm{v}}=G_{\mathrm{s}}$, the thick dashed curve (set B) goes down to the bold one (set C) and consists with the lattice result [1]. Thus, the PNJL calculations with set $\mathrm{C}$ can reproduce the lattice result [1] that $T_{\mathrm{c}}^{\sigma}$ coincides with $T_{\mathrm{c}}^{\Phi}$ within numerical errors in the entire region $0 \leq \theta \leq 2 \pi / 3$.
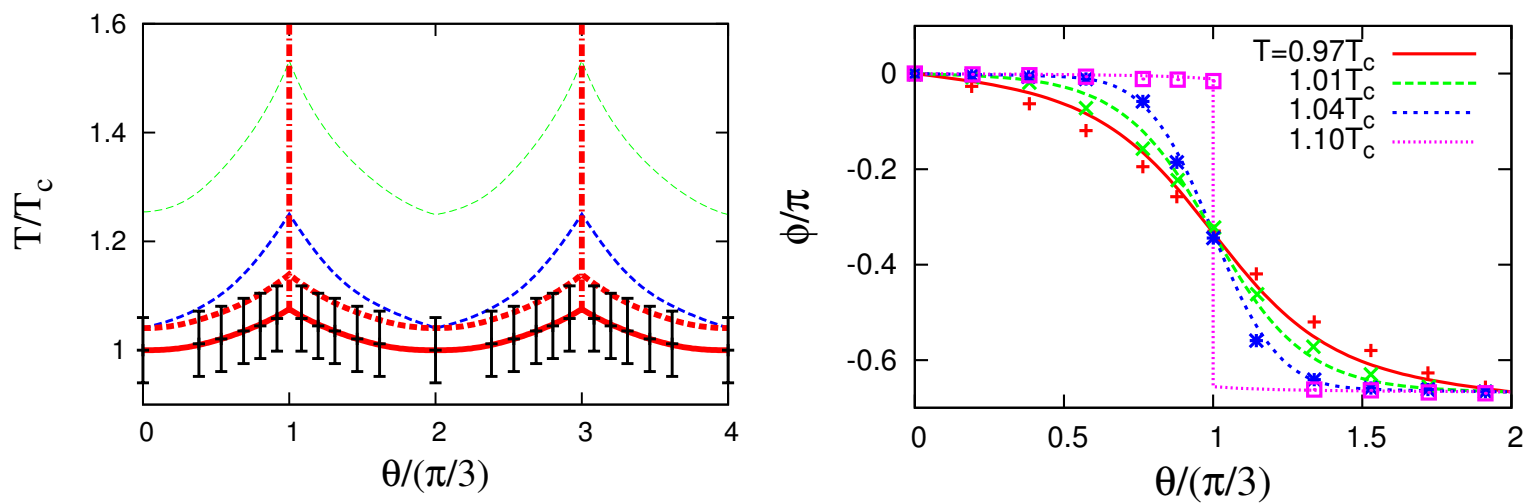

Figure 2: Left panel; Phase diagrams of the chiral phase transition in the imaginary chemical potential region calculated with three parameter sets are presented by dashed curves; thin, thick, and bold ones are results of the PNJL calculations with set A, B, and C, respectively. Lattice data [1] are shown with 10error that Tc has [41]. The deconfinement phase transition curve (bold solid curve) and the RW phase transition lines (bold dot-dashed lines) calculated with set $\mathrm{C}$ are also shown for comparison. Right panel; Phase of the Polyakov loop as a function of $\theta$ at various temperatures. Lattice data [1] are plotted by symbols. Curves represent results of PNJL calculations with set $\mathrm{A}$.

\section{Prediction of the phase diagram at real chemical potential}

Figures 3(a) and 3(b) represent the phase diagrams in the $\mu^{2}-T$ plane predicted by the PNJL calculations with parameter sets $\mathrm{A}$ and $\mathrm{C}$, respectively. On the solid curve between points $\mathrm{E}$ and $\mathrm{D}$, both the first-order chiral and deconfinement phase transitions take place simultaneously, and hence point $\mathrm{E}$ is the critical endpoint of these phase transitions. The dot-dashed curve moving up from point I represents the RW phase transition of first order, and then point $\mathrm{I}$ is the critical endpoint of the RW phase transition. The dashed curve between points $\mathrm{H}$ and $\mathrm{E}$ means the crossover chiral transition, while the long-dashed curve between points I and $\mathrm{E}$ does the crossover deconfinement phase transition. Point $F(G)$ is a crossing point between the dashed (longdashed) curve and the $\mu=0$ axis. Cross symbols with error bars indicate LQCD data [1].

Figure 3 (b) is most reliable, since the PNJL result with parameter sets $\mathrm{C}$ is consistent with the LQCD one [1] in the $\mu^{2}<0$ region. Comparing Figs. 3 (a) and 3 (b), one can see that the scalartype eight-quark interaction and the vector-type four-quark interaction give sizable effects on the 


\begin{tabular}{cccccc}
\hline set & D & E & F & H & I \\
\hline A & $(2.02,0.00)$ & $(1.84,0.72)$ & $(0.00,1.25)$ & $(i \pi / 3 \times 1.53,1.53)$ & $(i \pi / 3 \times 1.09,1.09)$ \\
\hline C & $(1.80,0.00)$ & $(1.51,0.72)$ & $(0.00,1.05)$ & $(i \pi / 3 \times 1.13,1.13)$ & $(i \pi / 3 \times 1.07,1.07)$ \\
\hline
\end{tabular}

Table 2: Positions of points D-I in $\mu$-T plane. The positions of these points are normalized as $\left(\mu / T_{\mathrm{c}}, T / T_{\mathrm{c}}\right)$ with $T_{\mathrm{c}}=173 \mathrm{MeV}$. Both set $\mathrm{A}$ and set $\mathrm{C}$ have the same position of point $\mathrm{G}$; $(0.00,1.00)$.

phase structure. In particular for the critical endpoint $\mathrm{E}$, the eight-quark interaction shifts point $\mathrm{E}$ to larger $\mathrm{T}$ and smaller $\mu$, and the vector-type interaction moves it in the opposite direction. Positions of points D-I are summarized in Table 2. The critical endpoint does not disappear in virtue of the eight-quark interaction, even if the vector-type interaction is taken into account.
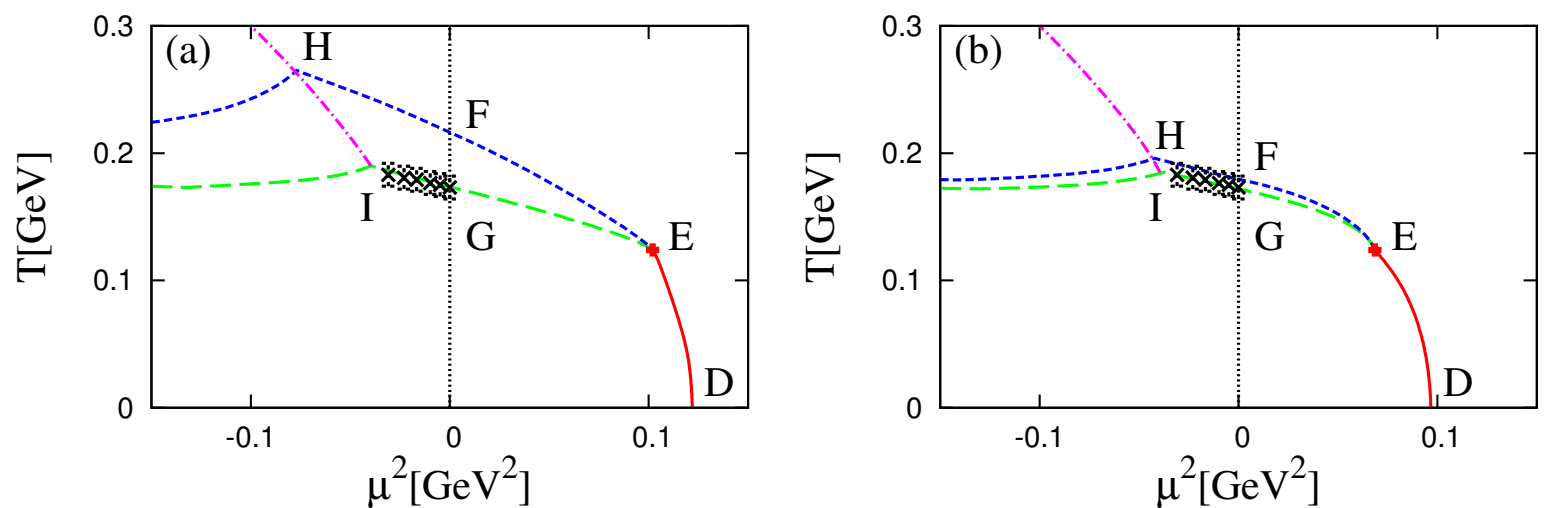

Figure 3: Phase diagram in the real and imaginary chemical potential region. Panels (a) and (b) are calculated with the parameter sets A and C, respectively. Cross symbols with error bars indicate the lattice data taken from Ref. [1]. Points D-I are explained in the text.

\section{References}

[1] P. de Forcrand and O. Philipsen, Nucl. Phys. B642, 290 (2002). L. K. Wu, X. Q. Luo, and H. S. Chen, Phys. Rev. D76, 034505 (2007).

[2] K. Fukushima, Phys. Lett. B 591, 277 (2004); Phys. Rev. D 77, 114028 (2008).

[3] S. Rößner, C. Ratti, and W. Weise, Phys. Rev. D 75, 034007 (2007).

[4] A. Roberge and N. Weiss, Nucl. Phys. B275, 734 (1986).

[5] Y. Sakai, K. Kashiwa, H. Kouno, and M. Yahiro, Phys. Rev. D 77, 051901(R) (2008); Phys. Rev. D 78, 036001 (2008); Phys. Rev. D 79, 096001 (2009); Y. Sakai, K. Kashiwa, H. Kouno, M. Matsuzaki, and M. Yahiro, Phys. Rev. D 78, 076007 (2008).

[6] F. Karsch, Lect. notes Phys. 583, 209 (2002); F. Karsch, E. Laermann, and A. Peikert, Nucl. Phys. B 605, 579 (2002); M. Kaczmarek and F. Zantow, Phys. Rev. D 71, 114510 (2005). 